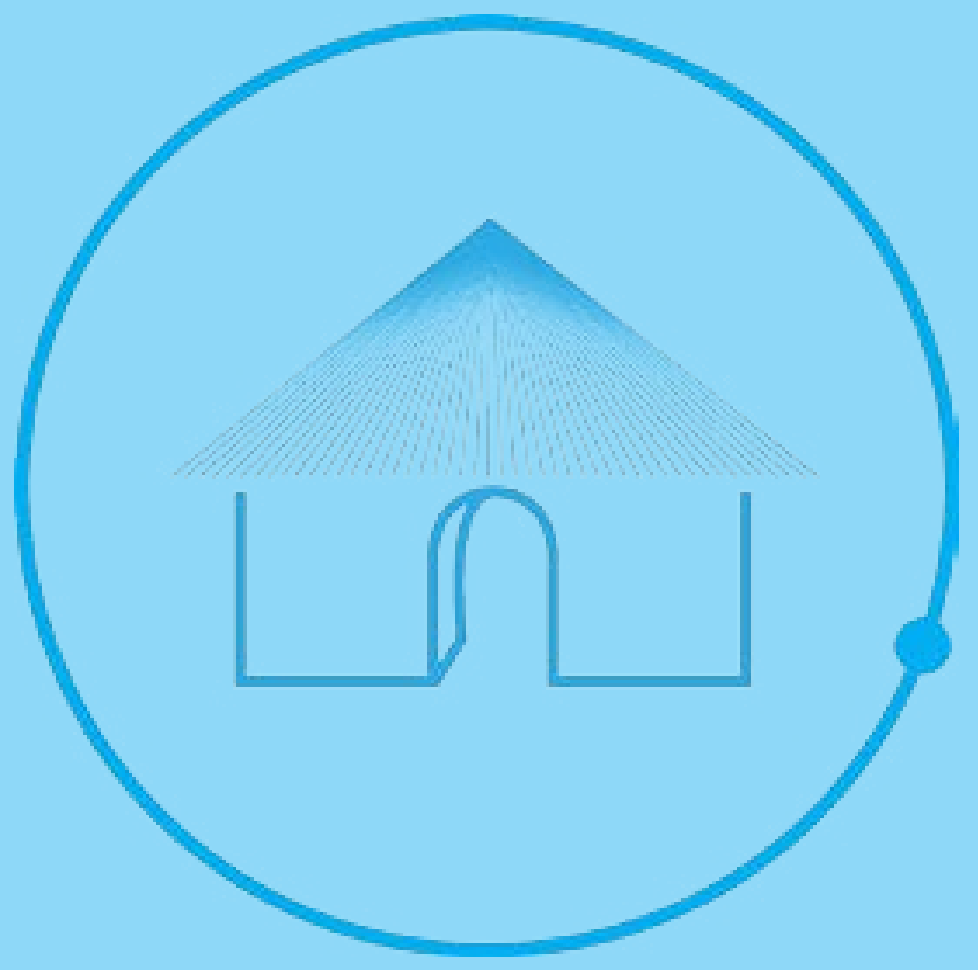

\title{
La investigación como estrategia pedagógica en la Guajira, desde una perspectiva de la inclusividad y diversidad como nuevo paradigma para el desarrollo de habilidades, destrezas y competencias
}

Educational research as a strategy in perspective of Guajira inclusiveness and diversity as a new paradigm for development of skills, skills and competence

Pesquisa educacional como estratégia na perspectiva da inclusão Guajira e diversidade como um novo paradigma para desenvolvimento de habilidades, capacidades e competências 


\section{Carlos Alfaro Camargo ${ }^{1}$}

1 Docente de la Universidad de La Guajira y del Liceo Moderno Madre Laura; Licenciado en Educación con énfasis en Matemáticas y física. Mg., en ciencias aplicadas, Física. Docente nominado al premio Compartir al maestro 2013-2014, vinculado al programa Ondas de Colciencias. Correo electrónico: docente_fisica@hotmail.com; docente_fisica@umiguajira.edu.co

Fecha de recepción: 23 de febrero de 2015 / Fecha de aprobración: 3 de abril de 2015.

\section{Resumen}

Todos somos conscientes de que una Colombia mejor parte del desarrollo y fomento de la educación, la ciencia y la tecnología. La investigación, como estrategia en el aula propone una visión diferente que, asumida desde el espectro de estrategia pedagógica en Ondas, permite una motivación hacia el aprendizaje y posibilita que el estudiante tenga acceso a múltiples escenarios de enseñanza, desarrollando habilidades, destrezas y competencias, que parten de su interés y del contexto en el que se inscribe, un aula que motiva es aquella en la que surgen las experiencias pedagógicas más exitosas del país.

\section{Palabras clave}

Diversidad, educación, formación inclusiva, investigación, trabajo colaborativo.

\section{Summary}

We are all aware that a better Colombia will start from the development and promotion of education, science and technology. Research as a strategy in the classroom offers a different vision, taken from the spectrum of pedagogical strategy stipulates Waves motivation to learning allows the student to access multiple scenarios in education, developing skills, abilities and skills, starting from interest thereon and the context in which is located a motivating classroom where the most successful educational experience in Colombia arise.

\section{Key words:}

Diversity, education, inclusive education, research, collaborative work.

\section{Resumo}

Estamos todos conscientes de que a Colômbia melhor, será iniciada a partir do esenvolvimento e da promoção da educação, ciência e tecnologia. A pesquisa como estratégia na sala de aula oferece uma visão diferente, tomada a partir do espectro de estratégia pedagógica estipula Waves motivação para a aprendizagem permite que o estudante para acessar vários cenários em educação, o desenvolvimento de habilidades, capacidades e competências, a partir de respectivos juros e do contexto em que está localizado a uma sala de aula motivando onde surgem a experiência educacional de maior sucesso na Colômbia.

Palavras chave:

Diversidade, educação, educação inclusiva, a investigação, o trabalho colaborativo. 


\section{Introducción}

$\mathrm{L}^{2}$ a creatividad, la capacidad de asombro, el poder de la innovación, la pasión por enseñar, la caridad, la humildad, la ternura, la empatía, son algunos elementos importantes en el arte de enseñar; la docencia es un apostolado que se debe irradiar en todos los sentidos y que, como docentes, nos exige empoderarnos a partir de esos principios básicos. Parte del éxito de la labor docente se centra en penetrar esa burbuja de cada estudiante, para, una vez dentro, conocer sus fortalezas, debilidades, sueños, temores e historia; lo que, desde mi experiencia como docente en la educación básica y media, llamaría conocer la naturaleza del niño "Acéptame como soy; solo así conocerás mi pequeña burbuja".

El arte de enseñar se debe centrar en los intereses de los niños, debemos apuntar al poder del aprendizaje, pero de uno guiado por el docente, en el que, para el niño, aprender sea sinónimo de divertido, una celebración de la vida, una exploración del potencial de cada uno y de la alegría de experimentar. Así, la investigación en Ondas es una fuente de experiencias y vivencias de aprendizajes, los niños y niñas encuentran vínculos fuertes de amistad, aprenden unos de otros, desarrollan su pensamiento científico y al tiempo son críticos, reflexivos y capaces de aceptar la opinión del otro, desde sus afinidades y diferencias.

Considerar la investigación como estrategia pedagógica es recrear ese espacio de inclusión, de diversidad; es hacer evidentes esos canales de la creatividad del docente, para convertir una pregunta del niño en una investigación; es devolverle al docente su pasión por enseñar, por potenciar las habilidades y destrezas de los niños y niñas, que están ávidos de conocimientos, convirtiendo la educación en un vehículo de capacitación, de oportunidades y de recreación, no de opresión.

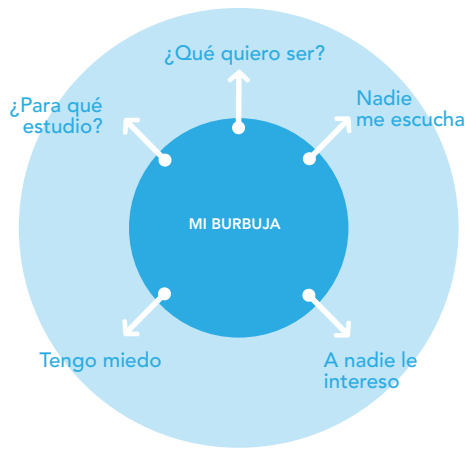

\section{Metodología}

\section{Comencemos por unir todas las burbujas}

Cuando el docente Ondas transforma el aula de clase en todo un laboratorio para el aprendizaje, convierte aquel espacio de cuatro paredes en el escenario propicio para que el niño (a) permita la entrada a su pequeña burbuja, entonces, desde adentro, el docente observa cuidadosamente que todas esas burbujas se unen en una gran burbuja, fluyen intereses, se conjugan temores, sueños, ideas del mundo, de política, moda, etc.; los niños expresan lo que esperan del mundo, piden que los ayudes a encontrar su lugar en la sociedad: "[...] la educación y el aprendizaje no pueden estar al margen de la sociedad en la que viven los que enseñan y aprenden” (Beltrán y Pérez, 2002, p. 233).

Cuando le das a conocer al niño que él es importante para ti, que sus preguntas e ideas son brillantes, captas toda su atención, estás en un momento sublime del acto pedagógico, es donde la Onda del conocimiento por fin ha penetrado en esa pequeña burbuja. La doctora Elaine Beauport afirma que: "En un mundo tan dinámico y de constante vibración, mundo cuántico donde todo es materia y energía, se concibe el cerebro y el cuerpo de los niños(a) como energía en movimiento continuo", esa energía, ese dinamismo es el que, a través de la investigación, aprendemos a canalizar los docentes Ondas desde el espectro de mundo que concibe el niño.

\section{El poder de la observación}

Durante los últimos años, la formación en la educación básica y media en Colombia ha encontrado un elemento fundamental: gracias al trabajo del maestro Ondas, ha comenzado a romper con el paradigma de la investigación como una actividad exclusiva de la educación superior:

\footnotetext{
Una línea metodológica que trata de dar salida al debate contemporáneo sobre la ciencia y su incidencia en la educación, implica usar la investigación para dinamizar procesos escolares y generar alternativas metodológicas para construir una escuela cercana a la configuración de un espíritu científico (Mejía y Manjarrez, 2013)
} 
Esta apreciación lleva a reflexionar sobre la importancia de cultivar en los niños y niñas una cultura en ciencia, tecnología e innovación, partiendo de nociones inherentes a su propio espíritu, como su capacidad de asombro frente a los fenómenos sociales y naturales. La investigación como estrategia pedagógica en Ondas, busca desarrollar dos principios importantes de la observación; el primero, que el niño(a) pueda recrear sus sentidos desde su proyecto, y el segundo, que la observación sea sistemática, es decir, que se incorpore al niño(a) como un hábito.

\section{La duda como factor decisivo a la hora de construir conocimientos}

La indagación permite al científico desprenderse de las explicaciones de otros para buscar las propias (Reyes, 2003). Así, otro aspecto importante en el desarrollo de la estrategia es la pregunta; la duda es uno de los ingredientes importantes en la generación de conocimientos, está matizada por la incertidumbre, que a la vez trae consigo la curiosidad, esta es potenciada por el docente Ondas a partir de múltiples iniciativas que luego se transforman en preguntas, solo una de ellas es considerada por el grupo como la pregunta clave para iniciar todo un proceso sistematizado de indagación y experimentación.

El docente Ondas se convierte así en un experto en la creación de espacios propicios para la generación de preguntas: “[...] la mayoría de las ideas iniciales son vagas y requieren analizarse con cuidado para que se transformen en planteamientos más precisos y estructurados" (Hernadez, R., Fernadez, C., y Baptista, P., 2010). Esta postura nos recuerda la forma como el Maestro Ondas canaliza cada una de las preguntas de los niños y la moldea hasta transformarla en una pregunta de investigación, recordemos que no hay preguntas bobas, ni respuestas definitivas, pero las preguntas siempre van más allá de la información, y luego se convierten en guías, en hilos conductores: "Para abrir estas nuevas vías es necesario replantear la creencia que establece que el conocimiento solo se produce desde el raciocinio que, de forma inductiva o deductiva, se relaciona con una base empírica" (Hernandez, 2007).

Al tiempo, el docente Ondas debe asegurarse de desarrollar en los niños(as) la habilidad de la Descripción, es decir, la forma como comunican, como sistematizan sus experiencias: "Describir, consta de una serie compleja de acciones, operaciones, de orden físico, mental y comunicativo" (Mantilla, 1997, p. 57). La descripción responde a esta exigencia, permite que el estudiantado haga una imagen de los pormenores del objeto que estudian.

\section{La evaluación desde Ondas}

El concepto de evaluación se relaciona con el aprendizaje de los niños(as); en nuestro sistema educativo se entiende como algo más que una simple medición de los productos y realizaciones. Es, ante todo, una práctica reflexiva propia del docente investigador Ondas, pues parte del interés del niño, de su forma de ver el mundo para, sistemáticamente, construir conocimientos. Cuando los niños socializan su experiencia, ese constructo social que nace después de todo un pensamiento colectivo, las miradas reflexivas permiten un empoderamiento que se manifiesta en el sentido de pertenencia por su escuela y su región. Maturana (2008), afirma que cada vez creamos un espacio de convivencia y reflexionamos en él, desde una mirada que analiza las consecuencias de lo que hacemos en él, puede surgir algo nuevo, un cambio de mentalidad, una mirada diferente del espacio escolar (p. 43), es así que, desde Ondas, la evaluación cumple las siguientes funciones:

a. Función orientadora: En la medida en que permite al docente conocer los intereses de los niños, contribuye en la elaboración de proyectos de investigación, centrando al estudiante en aspectos básicos que debe alcanzar para lograr propósitos colectivos. Esta función nos induce a la evaluación inicial, al diagnóstico y al pronóstico, pues se centra en situaciones reales, contextuales, que necesitan ser diagnosticadas; por el lado del pronóstico, permite hacerse a una idea inicial de trabajo, con un horizonte claro.

b. Función formativa: Desde esta función, la evaluación está orientada a la toma de medidas. Para hacerlo, se toma en cuenta la manera como cada estudiante se sitúa o toma parte de las actividades del grupo; su respeto por la opinión individual; la aceptación colectiva; las normas sociales; el respeto por la diferencia, y la naturaleza misma; y el fomento de una cultura de paz que está mediada por esa evaluación continua, permanente y por procesos. 
c. Función sumativa: Compuesta por la auto-evaluación, la co-evaluación y la hetero-evaluación, permite comprobar los resultados alcanzados y valorar el grado en el que se lograron. Desde el pensamiento colectivo, el grupo de investigación mide alcances, debilidades y fortalezas que luego se ajustan y contribuyen como insumos para la evaluación final.

d. Función social: Exige tomar como referencia criterios y objetivos trazados inicialmente, lo cual garantiza a todos los niños investigadores una experiencia significativa. Cuando se desarrollan competencias ciudadanas desde el proyecto de investigación, da como valor agregado el pleno empoderamiento de los niños sobre problemáticas sociales que pueden darse en sus escuelas, barrios, municipios y departamentos, desde un espectro crítico que contribuye en la búsqueda de sus soluciones.

e. Función de calidad: Permite abordar cambios e innovaciones en los programas educativos y acciones didácticas, basándose en rigurosas percepciones de la realidad, lo que contribuye, al optimizar la acción docente, a mejorar de la calidad de la enseñanza. En Ondas, uno de los aspectos motivacionales para los estudiantes es dar a conocer su trabajo, la socialización o la "perturbación de la ondas", término empleado desde el mismo programa para señalar los resultados: como el incremento en el sentido de pertenencia por su región y por su escuela; el respeto para con los docentes y una serie de aspectos propios del proceso de formación, que van, desde el desarrollo de competencias comunicativas y científicas, hasta las ciudadanas.

\section{Técnicas e instrumentos de evaluación del maestro Ondas}

En Ondas se emplean estrategias de evaluación metacognitivas a través de instrumentos como los diarios reflexivos, las bitácoras, los portafolios, la autorregulación del aprendizaje mediante la elaboración de mapas conceptuales, la auto-observación, el uso de matrices de evaluación y roles de moderador y relator etc. (Rodriguez, J., y Tejedor, F., 1996). Es importante resaltar si los estudiantes logran traducir nueva información a su propia terminología, es decir, si generan ejemplos concretos desde su propia experiencia, que puede traducirse a un aprendizaje no solo situado, sino significativo.

"El maestro activo necesita de otras cualidades que no son las que se reclamaban antes de él. Ya no necesita tener autoridad, pues ya no hay autoridad que ejercer, en tanto que sus estudiantes actúan autónomamente y su rol es de apoyo" (Reyes, 2003). Desde esta mirada, la evaluación se convierte en un instrumento muy valioso para que el docente aprenda a evaluar y brindar indicadores del aprendizaje de sus estudiantes: "Hoy el aprendizaje y la evaluación deben tomar en consideración el desarrollo del propio estudiante, es decir, sus expectativas, su nivel inicial, sus estilos de aprendizaje, sus ritmos e intereses [...] sus necesidades y proyección futura (Bordas Cabrera, 2001). El reto de la evaluación es considerar cómo debe plantearse para ser pertinente con las diferentes posturas pedagógicas, en favor de un aprendizaje significativo y respetuoso con las particularidades individuales y culturales de los niños y niñas.

\section{Mi experiencia en el aula}

Estar vinculado por más de 10 años al programa Ondas de Colciencias me ha permitido conocer y apostarle a la investigación como estrategia pedagógica. Me empoderé de los lineamientos del programa y pude entender y valorar las preguntas de los niños y niñas; asumí la estrategia metodológica, es decir, la investigación como una herramienta poderosa que acompaña nuestro quehacer pedagógico; por último, adopté la parte más sublime de lo pedagógico: la sistematización, que no solo permite organizar nuestra experiencia, sino que facilita a los niños ser los actores principales del proceso.

Según Martínez (2009), una investigación tiene un alto nivel de validez si al observar, medir o apreciar una realidad, se observa, mide o aprecia esa realidad. Es decir, a pesar de que el conocimiento ha sido develado con anterioridad, y de que el niño(a) lo ignora, éste aparece frente a él como un nuevo constructo: "[...] el aprendizaje significativo se caracteriza por la interacción entre el nuevo conocimiento y el conocimiento previo" (Moreira, 2005). Lo previo consistirá en la información consultada por los niños y niñas al momento de realizar su investigación; en 
este sentido, no podemos perder de vista el sentido estricto de la investigación: la creación de una nueva realidad ante los estudiantes, eso es lo que llamaríamos un aprendizaje significativo.

Como docente acompañe más de 20 proyectos de investigación de los niños en el programa Ondas de Colciencias, de los cuales referenciaré solo cuatro de ellos. Puedo afirmar lo importante que ha sido el programa en el desarrollo de mi vida profesional, pues ha significado exigirme, abrir una y otra vez las viejas posturas pedagógicas, romper una y mil veces las viejas estructuras para tratar de darles nuevas formas, nuevos métodos.

El proyecto Ondas termina convenciendo de lo importante de su metodología, que envuelve y dirige el proceso de enseñanza y aprendizaje; obviamente no existen recetas para asegurar una práctica docente eficaz, pues los contenidos y objetivos varían, cada grupo y estudiante difieren en su forma de aprender y las circunstancias y situaciones siempre son diferentes, sin contar con las características personales de cada maestro que media el proceso: "Mediación es un elemento inherente e insustituible en la facilitación del Proceso de enseñanza y aprendizaje" (Linares, s.f).

Por tanto, analizar desde el proyecto Ondas la pedagogía fundamentada en la investigación, significa que es necesario contemplar un cambio en la idea de maestro y de su rol como orientador del aprendizaje; el docente debe dejar a un lado el acto repetitivo de la enseñanza mecanizada, para pasar a una reflexión diaria de su quehacer, debe permitir la producción de saberes a través de un enfoque teórico-práctico donde la enseñanza colaborativa, de indagación, problemática y situada construya escuelas nuevas, flexibles, cambiantes, generadoras de nuevos saberes acordes con las necesidades locales.

\section{Teatro-Ciencia}

Uno de los proyectos de investigación que desarrollé con los niños y niñas durante mi proceso en el programa Ondas fue el Teatro-Ciencia, llevado a cabo con el grado séptimo de la Institución Educativa Norberto Iguarán Webber de la ciudad de Maicao, en La Guajira. Utilizamos el teatro para dramatizar la vida de muchos científicos, sus aportes más relevantes; durante el trabajo los estudiantes hacían noticieros, entrevistas a científicos famosos, poesías y cuentos. La clase se hacía dinámica gracias a las consultas previas de los estudiantes; la personificación colectiva, el guión y las escenas, permitían a los niños desarrollar com- petencias comunicativas, científicas, teatrales y hasta ciudadanas.

En cuanto al uso de lo teatral como instrumento pedagógico, maravilloso y útil, es posible afirmar, siguiendo las ideas de Cañas (1992), que el Teatro abre como ninguno las puertas de la imaginación y de la creatividad, que libera inhibiciones, socializa conductas y, junto al juego, se convierte en una herramienta pedagógica poderosa que reproduce y enseña. El teatro, como herramienta pedagógica, requiere de la comunicación para provocar y activar los mecanismos de aprendizaje; así, la clase se impregna de un ambiente lúdico y permite a cada estudiante desarrollar sus propias estrategias de aprendizaje mientras se divierte.

También me vi en la posibilidad de realizar un proyecto social: "Wuin Yarrutsu”, que en Wayuunaiki, dialecto de los indígenas Wayuu, significa "Aguas negras"; en él se hizo evidente la participación de la escuela como entidad que actúa en favor de solucionar problemas ambientales del entorno. Para abordarlo, es necesario reconocer la importancia que el sistema educativo nacional da a la implementación de los Proyectos Educativos Ambientales (MEN, 2005); esto llevó a la creación de los grupos PRAE en las instituciones educativas.

Así, desde una visión integral del medio ambiente y de sus problemáticas, se dio paso a la construcción, en la institución, de procesos de formación que permitieran comprender los problemas y reconocer y construir vías para desarrollar acciones concretas que redundaran en beneficio de la sostenibilidad del ambiente. Estas vías se deben ver desde lo cognitivo, pero también desde lo axiológico, para llegar a una proyección pertinente.

\section{"Wuin Yarrutsu"}

El proyecto de investigación "Wuin Yarrutsu” se ejecutó a partir de la alianza entre dos instituciones educativas: El Liceo Moderno Madre Laura, de carácter privado, y la Institución Educativa $N^{\circ} .1$ de la ciudad de Maicao. Fue patrocinado por el programa Ondas y permitió que los estudiantes investigaran acerca de los estragos ocasionados por la laguna de oxidación del Municipio de Maicao en las comunidades de Limoncito, Naranjito y Laguna, consideradas resguardos indígenas. Para hacerlo fue necesario seguir los principios de Romero (2006), cuando afirma que las escuelas necesitan cambiar su dimensión pedagógica y sus aspectos organizativos, considerando que a pesar de que son el foco de la unidad de cambio, no pueden hacerlo solas. 
El problema se daba, por una parte, debido a la falta de mantenimiento de la laguna y, por otra, a su ubicación; las fallas en la planta de bombeo de aguas negras afectaban el consumo de agua de los animales, hecho que se reflejaba en el comercio de carnes en el mercado público, pues cuando consumían estas aguas morían. Es importante destacar que muchos de los estudiantes Wayuu de la Institución Educativa $N^{\circ} .1$ eran habitantes de este sector y, en épocas de invierno, se veían afectados por su constante contacto con este medio.

\section{El Objetivo}

Conocer el impacto ambiental de la creación de la laguna de oxidación del Municipio de Maicao en las comunidades del Limoncito, Laguna, Naranjitos y barrios aledaños.

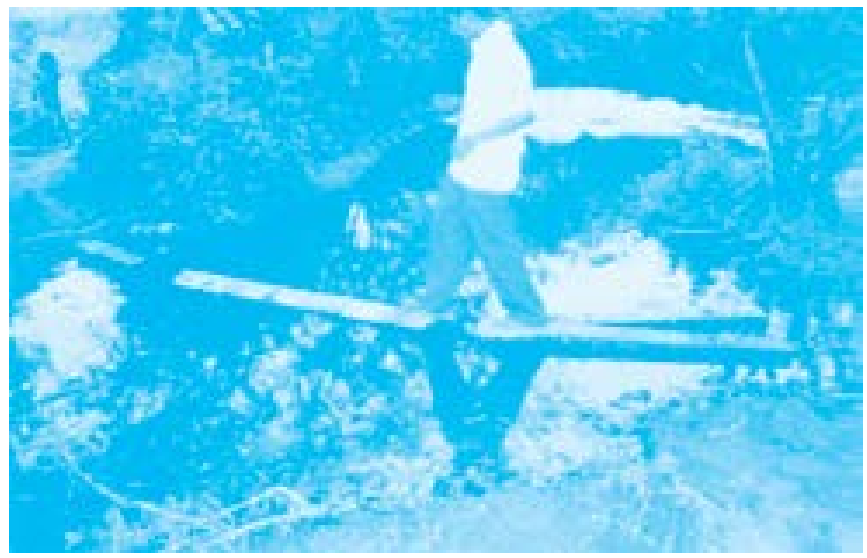

Figura 1. Estudiante de la Institución Educativa $N^{\circ} 1$ dirigiéndose a su escuela (Febrero, 2005)

Nota. Fuente, Carlos Alfaro Camargo

El Instituto Colombiano de la Reforma Agraria afirma que los resguardos indígenas: "[...] estarán dirigidos a facilitar el cumplimiento de la función social y ecológica de la propiedad por parte de las comunidades, conforme a sus usos o costumbres, a la preservación del grupo étnico y al mejoramiento de la calidad de vida de sus integrantes" (Ley 160, 2004, Parágrafo 3). El conocimiento de la norma permitió que los niños se dieran cuenta de que los indígenas de la zona estaban siendo afectados por la cercanía de la laguna a sus rancherías y de la responsabi- lidad del Estado de velar por mejorar la calidad de vida de este grupo étnico. A partir de este descubrimiento realizaron visitas de campo, entrevistas y encuestas a miembros de esas comunidades para diagnosticar la situación.

\section{Impacto en la comunidad}

- Se contribuyó al mejoramiento de la calidad de vida de las comunidades del Limoncito, Laguna y Naranjito.

- $\quad$ Se proyectó a las dos instituciones como entidades capaces de desarrollar trabajos que le reporten un bien social a sus respectivas comunidades.

- Se dio a conocer la problemática a través de un foro municipal donde participaron las autoridades competentes y la Universidad de la Guajira, exponiendo un tema relacionado con el manejo de residuos sólidos y aguas residuales, y el diseño e implementación de lagunas de oxidación.

- Se repartieron más de 80 mercados.

- Se regaló ropa usada a los indígenas y material de primeros auxilios.

- Se denunció el caso ante el Ministerio de Medio Ambiente y Desarrollo Territorial.

- Se tocaron muchas embajadas solicitando ayuda para estas comunidades.

- Socialización de la experiencia en Expociencia Expotecnologia 2005 .

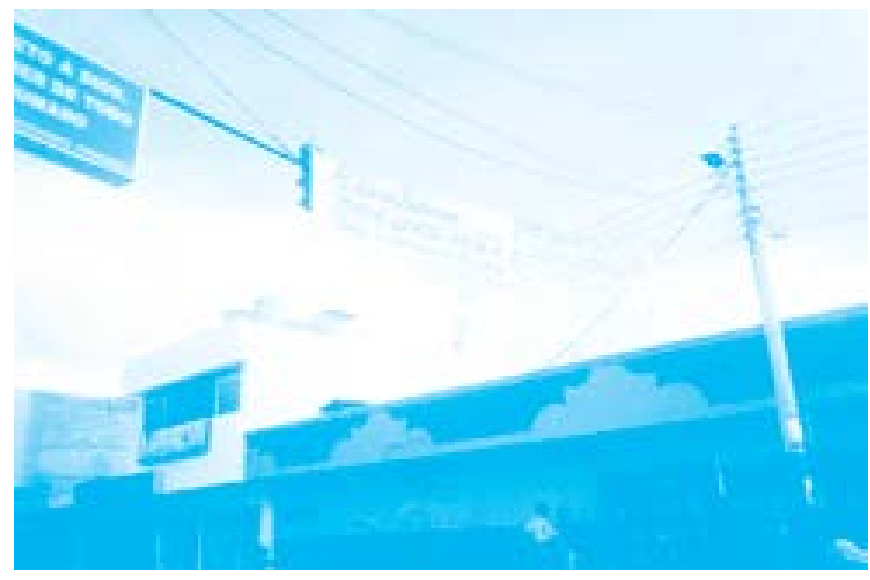


Figura 2. Convocatoria al primer Foro Ambiental (Junio 2005).

Nota. Fuente, Carlos Alfaro Camargo

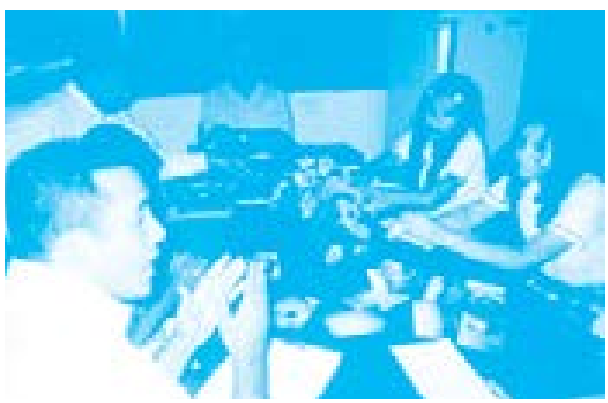

Figura 3. Mesa de trabajo con docentes de la Universidad de la Guajira (Abril, 2005)

Nota. Fuente, Carlos Alfaro Camargo

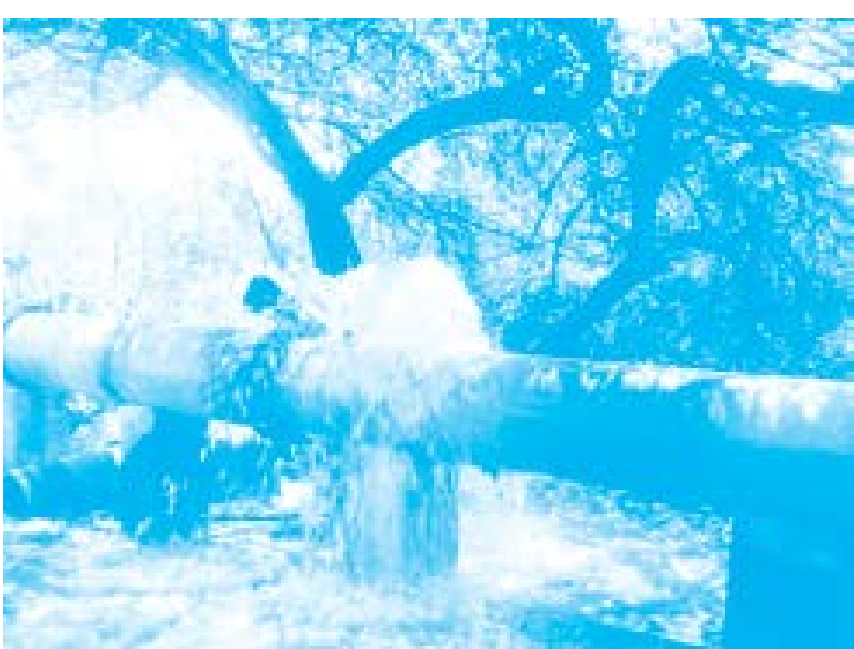

Figura 4. Aguas residuales en pésimo estado (Marzo, 2005) Nota. Fuente, Carlos Alfaro Camargo

La etnoastronomia: una manera de plantear y resolver problemas cotidianos desde la investigacion en un contexto histórico

La etnoastronomía fue un proyecto de investigación que se transformó en toda una estrategia pedagógica y fue nominado, gracias a la participación de un gran número de maestros, al Premio Compartir al Maestro 2014. La situación problemática giró alrededor de hacer que los estudiantes Wayuü participaran en clase, mientras se facilitaba a los estudiantes no Wayuü la posibilidad de conocer más de la cultura indígena, para, al tiempo, motivar a toda la clase al estudio de la astronomía; es necesario anotar que, debido a su condición cultural, los indígenas son muy reservados y herméticos en las escuelas.

Los estudiantes "alijunas" (no Wayuü) siempre se han reído cuando escuchan a los indígenas debido al género que utilizan para nombrar algunas cosas; por ejemplo, para un Wayuü la luna es un ente masculino, no dicen "la luna" sino "el luna"; así, se ha llegado a afirmar que no hablan bien el castellano. En consecuencia, a los miembros de la comunidad les da pena hablar en Wayunaiki (dialecto Wayuü) en presencia de un "alijuna” o, en el peor de los casos, niegan su condición indígena por lo que yo llamaría "vergüenza étnica"; esta situación hace que su participación en las clases sea baja y ha fomentado una pérdida de sus valores culturales.

¿Cómo lograr que los estudiantes del bachillerato se motiven?; ¿cómo lograr que los estudiantes Wayuü participen y los alijunas respeten sus valores culturales?; ¿cómo despertar en los chicos ese espíritu investigador?; ¿cómo conseguir que los estudiantes no Wayuü empezaran a conocer la cultura Wayuü, a valorarla y respetarla? Este fenómeno se presentaba en las dos escuelas donde he venido trabajando: el colegio privado Liceo Moderno Madre Laura y la Institución Educativa Nº.1, de carácter oficial.

\section{Estrategia pedagógica}

La estrategia pedagógica fue desarrollada en dos etapas: la primera (consulta) consistió en recomendar a los estudiantes una consulta sobre el origen de la astronomía, su importancia en el desarrollo social, cultural y económico en culturas como la Azteca, Maya, Inca, Griega y Egipcia, y, por supuesto, Wayuü, mientras, al tiempo, debían investigar las costumbres Wayuü, para lo cual se realizaron algunos debates con estudiantes y docentes del área de ciencias sociales y naturales (física); la segunda (contacto directo), consistió en visitar algunas rancherías (visita de campo) e interactuar con ancianos Wayuü; en este ejercicio no solo los ancianos se mostraron interesados, sino que muchos niños indígenas pusieron a disposición algunas rancherías de sus abuelos y parientes. 
En este contexto, se dio participación a los padres de familia, contando con su compañía en las salidas de campo (previa reunión); pensando que de esa manera lograríamos obtener algunos elementos para la investigación (grabadora periodista, filmadora, cámara fotográfica, binoculares, mapas celestes, entre otros). Fue necesario enfrentar el hecho de que pocos ancianos Wayuü hablan castellano y son muy herméticos con los alijunas; la solución fue involucrar a los niños indígenas, pues en su mayoría hablan perfectamente el Wayunaki y entienden el castellano. Socializamos la idea con los estudiantes del grado $10^{\circ}$ de la escuela oficial y estuvieron de acuerdo.

\section{Logros}

- Motivación de los estudiantes.

- Apropiación del método científico.

- Fortalecimiento de la capacidad de investigación de los estudiantes.

- Se conoció un poco más la etnia Wayuü, en especial algunas costumbres desconocidas que desarrollaron respeto hacia sus valores culturales.

- Integración de otros docentes en la estrategia.

- Interés por la lectura de (poesías, cuentos, mitos y leyendas Wayuü).

- Creación de un texto didáctico sobre los hallazgos de nuestra investigación.

- Creación del departamento de investigación de la escuela.

- Socialización de la investigación en diversos encuentros de experiencias significativas a nivel nacional.

- Reunión permanente ante un evento astronómico (eclipse, cometa etc.).

\section{La evaluación}

Para realizar el proceso de evaluación se tomaron en cuenta cuatro aspectos:

- Exploratorio: Confrontación entre saberes previos y encontrados.

- Constructivo: Promover la investigación y proporcionar habilidades comunicativas.
- Reflexiva: Etapa de control de la información y validez del concepto.

- Interdisciplinariedad: Las áreas de ciencias sociales, lengua castellana y ciencias naturales evalúan los procesos, desempeños y competencias de cada estudiante; para ello cada docente realiza un proceso de seguimiento y evaluación durante una hora de lectura estipulada por la institución, revisando la construcción de cuentos, poesías y leyendas que relacionan la investigación e involucrando los componentes procedimentales, conceptuales y socio-afectivos.

En el trabajo de campo los estudiantes presentan un informe escrito de la actividad, donde los docentes del área de lengua castellana, filosofía y física, evalúan aspectos como: expresión escrita, ortografía y gramática, organización del trabajo y el resumen o síntesis del mismo trabajo. Para las exposiciones de física (en lengua castellana o Wayuunaiki) algunas matrices de evaluación contienen aspectos como: manejo del público, tiempo estipulado, dominio del tema, timbre de voz y ayudas didácticas. En cuanto a los procesos de auto-evaluación de trabajos grupales, se consideraron aspectos como: el respeto por las opiniones de los compañeros, los aportes significativos al grupo, movilización de información confiable a través de páginas web, el cumplimiento de las tareas asignadas por el grupo y la participación en puesta al día del blog del grupo.

Para la evaluación docente los estudiantes consideraron los siguientes aspectos: acompañamiento constante a los procesos de movilización de información, directrices claras impartidas por el docente al asignar algún trabajo escrito, dominio de los temas propuestos por el estudiante cuando pide asesoría. En los componentes de la auto-evaluación el estudiante se enfrenta a preguntas como: si se sintió bien al momento de dar a conocer sus ideas, si sus esfuerzos se notaron y cuáles fueron los aspectos más relevantes de su aprendizaje.

\section{Resultados}

Los estudiantes alijunas han comprendido y conocido más profundamente la cultura Wayuu; los niños Wayuu realizan las exposiciones en física en su lengua materna; en las dos instituciones se elevó el promedio de física en las pruebas de estado, 
se recibió en dos ocasiones la distinción Andrés Bello en física. La propuesta hizo parte de Expociencia, Expotecnologia 2005 y fuimos invitados por la UNAD (Universidad Nacional Abierta y a Distancia) para socializar nuestro trabajo en Santa Marta y Barranquilla (Combarranquilla). Se contó con el apoyo de la Alcaldesa de Maicao para comprar 360 ejemplares de Etnoastronomía Wayü̈ y otras, que fueron obsequios para el Día del Maestro; el lanzamiento del texto se realizó en las instalaciones del Liceo Moderno Madre Laura y contó con la asistencia de escritores y pintores guajiros en el marco del cumpleaños de Maicao. Hemos sido ponentes en los congresos de astronomía en la Universidad de la Guajira 2012 y ganadores en el concurso Colombia vive la ciencia, vive la astronomía, en Ibagué 2013.

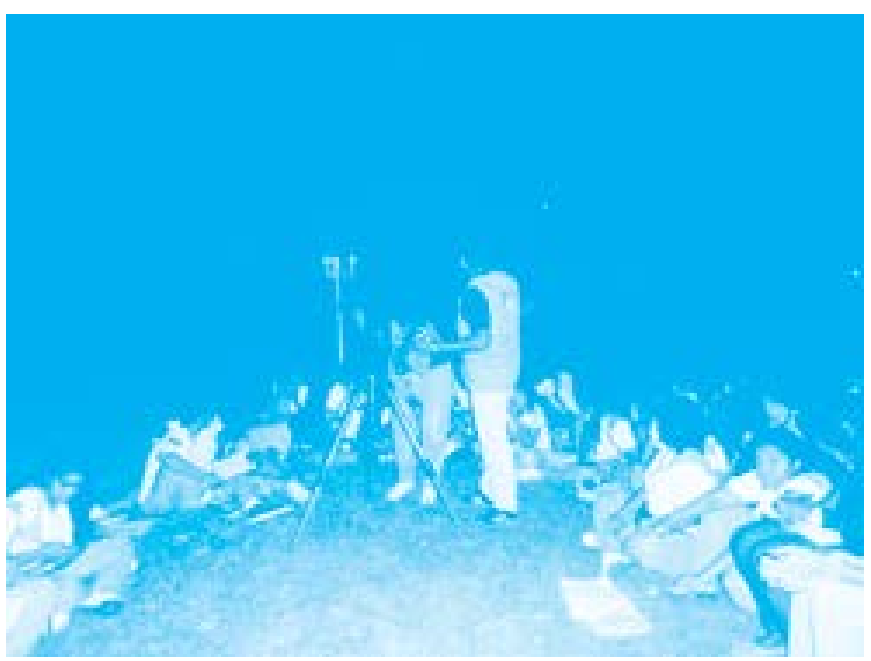

Figura 5. Actividad Astronómica de campo (2013) Nota. Fuente, Carlos Alfaro Camargo

\section{Proyecto Gliricidia Sepium (Mata-ratón)}

Durante el ejercicio de la docencia seguramente nos ha tomado por sorpresa alguna pregunta; sin embargo, ya sea por la interpretación sobre un fenómeno en particular o sobre algún acontecimiento pasado: "El método para aclarar la duda, sería la vía que facilite el descubrimiento de conocimientos seguros y confiables para darle solución a los problemas que la vida nos plantea" (Martinez, 2009, p. 161). Es decir que la enseñanza enfocada en la investigación toma múltiples caminos y hace énfasis en hechos particulares. Por ello, Mejia, R., y Manjarrez, M. (2013) afirman que para resolver el debate contemporáneo sobre la ciencia y su incidencia en la educación, es necesario usar la investigación para dinamizar los procesos escolares y generar alternativas metodológicas, esto con el objetivo de construir una escuela cercana a la configuración de un espíritu científico.

Un día los niños del grado octavo de la Institución Educativa $\mathrm{N}^{\circ} .1$ me abordaron para preguntar acerca de la planta conocida en la región como Mata-Ratón, querían saber si servía para matar ratones o ratas. En ese momento se presentaba un problema en el colegio: las personas que habitaban los alrededores compraban raticidas y este remedio no solo acababa con ratones y ratas, sino con los gatos, ya que luego de envenenarlas las tiraban en la parte posterior de la escuela, por supuesto los gatos se comían los restos y morían también, causando un problema ambiental.

Los niños encuestaron a toda la comunidad que habitaba los alrededores de la escuela para conocer la cantidad de personas que utilizaban raticidas y cuántos tenían gatos en sus casas; tomaron 8 ratas en cautiverio para las pruebas de laboratorio y extrajeron un concentrado de la planta Glirisidia Sepium (Mata-ratón). El producto fue aplicado a la población de ratas y, cuando empezaron a morir, se suspendió la investigación porque los niños se encariñaron con los roedores.

El Mata-ratón (Gliricidia Sepium) es una leguminosa arbórea que crece desde México hasta la parte norte de Suramérica, encontrándose ampliamente distribuida en las regiones tropicales. Es frecuente encontrarla en diferentes zonas del país en forma de cercas vivas y cuenta con gran cantidad de usos: siendo utilizado como sombrío, leña, madera y como soporte para algunos cultivos, además es útil como abono verde y como recuperador de suelos degradados (Abad, s.f.). Recientemente se ha planteado utilizarla como alimento para animales, pues es una alternativa interesante como fuente de proteínas en la dieta de rumiantes. Con este fin se han realizado siembras intensivas, dirigidas a la producción de biomasa para alimentar animales en confinamiento o como suplemento al pastoreo. 


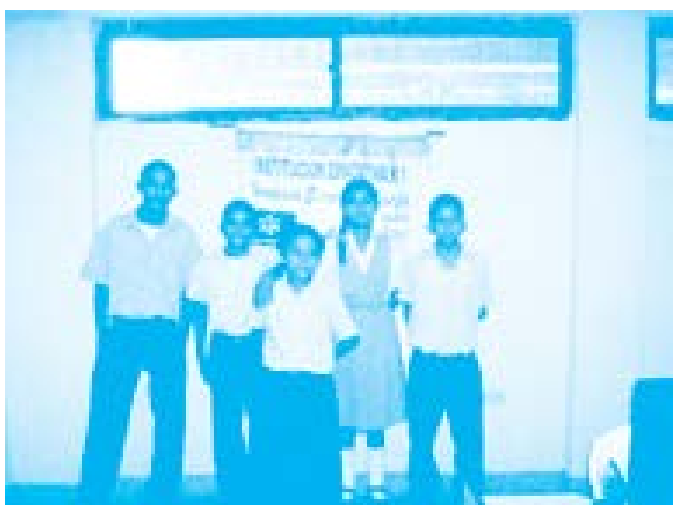

Figura 6. Estudiantes que participaron en la investigación (2008)

\section{Nota. Fuente, Carlos Alfaro Camargo}

\section{Objetivo general}

Comprobar si la planta (Gliricidia Sepium) conocida como Mata-ratón sirve para matar ratas o ratones.

\section{Impacto esperado en la comunidad}

- Concienciar a las personas para que eviten el uso de raticidas.

- Crear conciencia ecológica para que no se arrojen ratas muertas a la calle.

- Motivar aún más a los estudiantes por las clases de ciencia.

- Proteger a la población de gatos domésticos.

Resultados

Incentivar la protección del medio ambiente.

- Se demostró la efectividad de la Gliricidia Sepium como mata ratas.

- Se realizó un producto capaz de exterminar ratas y ratones.

- Se comprobó que para hacer ciencia no es necesario tener un laboratorio completo en nuestra escuela.

- Se dio a conocer el peligro de las ratas para el ser humano.
- Se logró vender la idea a la comunidad aledaña de que el mejor raticida es un gato.

- Se ha creado una conciencia ecológica en el grupo de investigadores.

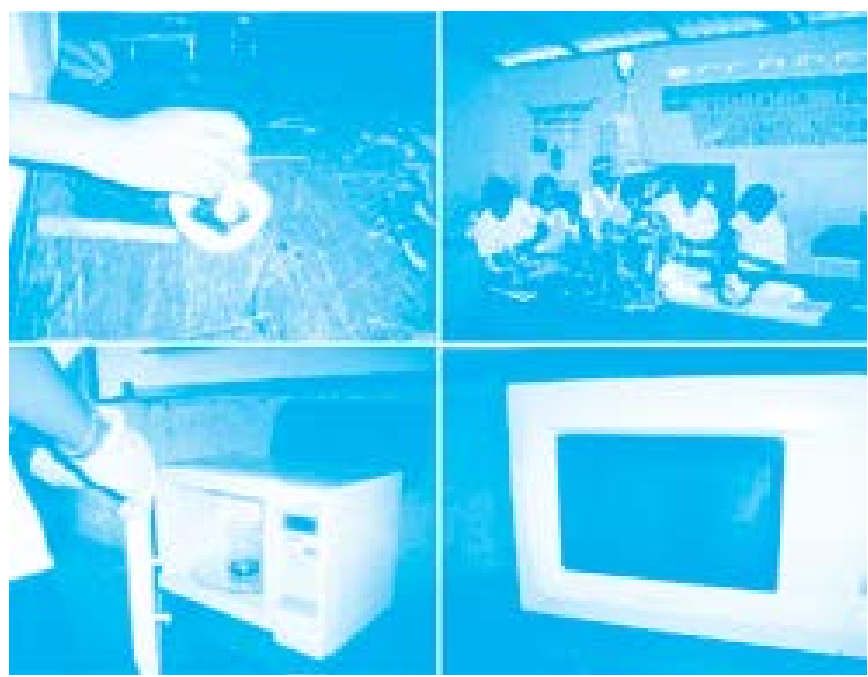

Figura 7. Estudiantes extrayendo concentrado de las hojas y médula de la planta (2009)

Nota. Fuente, Carlos Alfaro Camargo
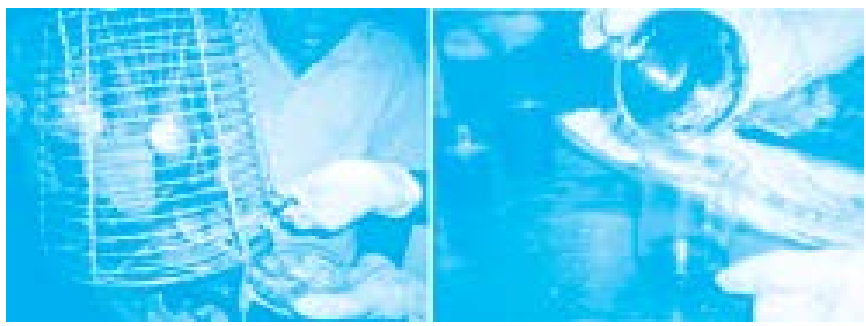

Figura 8. Estudiantes alimentando a las ratas con el concentrado (2008)

Nota. Fuente, Carlos Alfaro Camargo 


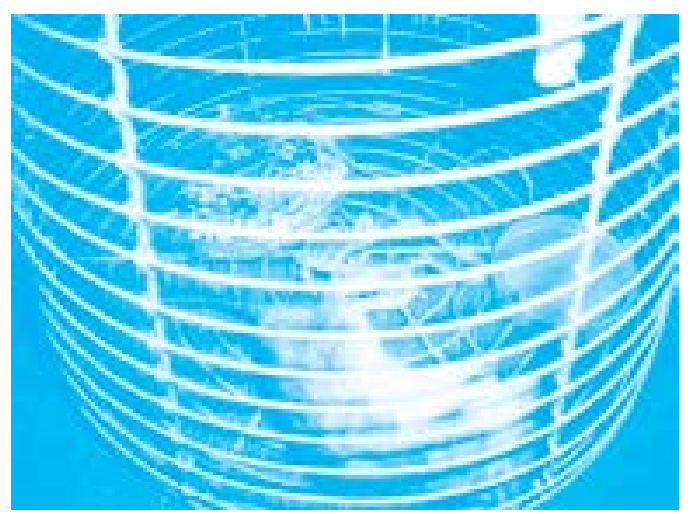

Figura 9. Efectos del Glirisidia Sepium en roedores (2008) Nota. Fuente, Carlos Alfaro Camargo

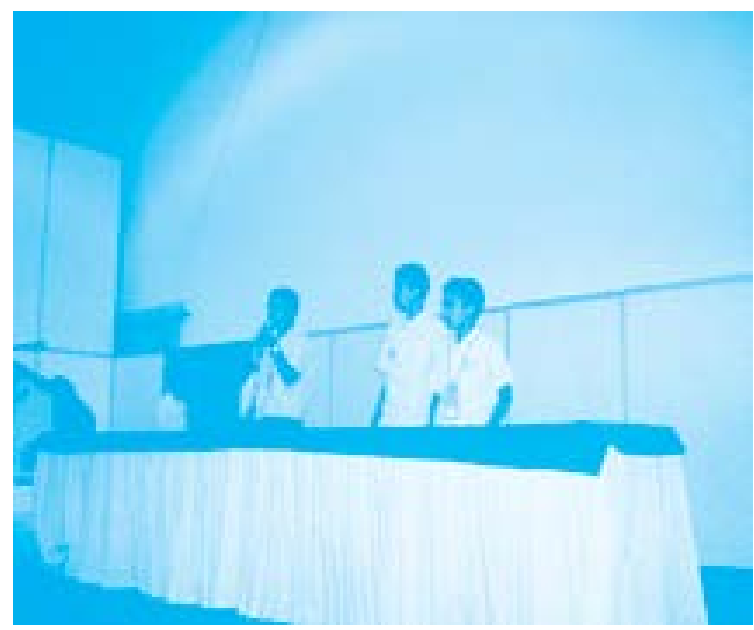

Figura 10. Socialización del proyecto en Expociencia Expotecnologia (2009)

Nota. Fuente, Carlos Alfaro Camargo

\section{Conclusiones}

La conformación del grupo de investigación en el programa Ondas se puede interpretar como el comienzo de un proceso de aprendizaje colaborativo; es una apuesta para romper con los viejos paradigmas de la educación, y ha propiciado una práctica formativa, situada, significativa y propositiva que enriquece a docentes y estudiantes, permitiendo a los niños investigadores escuchar, compartir, dialogar, reflexionar y aprender, llevándolos a la transformación de su entorno desde cambios significativos basados en el respeto a sí mismo y a los demás. 


\section{Referencias}

Abad, G. (s.f.). El mataratón. Obtenido el 12 de Octubre de 2007, desde http://www.corpoica.org. co/sitioWeb/Archivos/Publicaciones/Matarraton.PDF

Alfaro, C. (s.f.). Camino a la escuela. Maicao: Institucion Educativa $\mathrm{N}^{\circ} .1$.

Beltrán, J., y Pérez, F. (2002). El rol del profesor. En Cela, U. C. El profesor. Madrid: Espasa, p. 440.

Bordas Cabrera, M. (2001). Estrategia de evaluacion de los aprendizajes centrados en el proceso. Revista española de pedagogía. Obtenido el 01 de Agosto de 2014, desde http:// cmapspublic3.ih mc.us/rid=1GLSW84JS-WYZWX0-H40/Evaluaci\%C3\%83\% C6\%92\%C3\%82\%C2\%B3n\%20de1\%20Proceso $\% 20 \mathrm{de} \% 20$ Aprendizaje.pdf

Colciencias. (s.f.). Caja de herramientas. Obtenido el 3 de Agosto de 2014, desde http://www.colciencias.gov.co/sites/default/files/ Ondasfinal/libros/cat3/sub1/index.html\#/1/zoomed

Cañas, J. (1992). Didáctica de la expresión dramática: una aproximación a la dinámica teatral en el aula. Barcelona: Octaedro.

Hernández, R., Fernández, C., y Baptista, P. (2010). Metodología de la investigación. Lima: McGrawHill.

Hernández, F. (2007). La investigación basada en las Artes. Propuesta para repensar la investigación en educación. Educatio Siglo XXI. No. 26, pp. 85-118.
Linares, D. (s.f.). Facilitar y mediar aprendizajes. Ediciones 24.

Manjarréz, et al. (2007). La investigacion en el mundo del/la maestra(o). La investigación como estrategia pedagógica. Bogotá: Edeco, p. 88 .

Mantilla C. W. (1997). El cientifico y la ciencia. Bogotá: Ediciones Hispanoamericana.

Martínez, M. (2009). La nueva ciencia. México: Trillas.

Maturana, H. (2008). El sentido de lo humano. Buenos Aires: Granica.

Mejía, R., y Manjarréz, M. (2013). La investigación como estrategia pedagógica, una propuesta del Sur. Bogotá: Desde Abajo.

MEN. (2005). ABC de los Proyectos Educativos Escolares-PRAE. Obtenido el 20 de Enero de 2015, desde: http://www.colombiaaprende.edu.co/html/mediateca/1607/article-81637.html

Ministerio de Agricultura. (2004). Ley 160 de 1994. Obtenido el 20 de Febrero de 2015, desde http://www.incoder.gov.co/documentos/Ley160_2004.pdf

Moreira, M. (2005). Aprendizaje Significativo Crítico. Obtenido el 20 de Diciembre de 2014, desde file://D:/Bibliotecas/Descargas/ Dialnet-AprendizajeSignificativoCritico-1340902.pdf 
Reyes, E. (2003). Didácticas activas. En Zubiría, M. D. Enfoques pedagógicos y didácticos contemporáneos. Bogotá: FiPc Alberto Merani, p. 337.

Rodríguez, J., y Tejedor, F. (1996). Evaluación de los aprendizajes de los alumnos. Salamanca: Ilustrada.

Romero, C. (2006). La escuela media en la sociedad del conocimiento. Buenos Aires: Noveduc. 